\title{
制造系统可靠性分析的框架与动力学机制*
}

\author{
孔繁森 $1,2,3$ \\ (1. 数字孪生智能工厂规划设计吉林省重点实验室 长春 130022 ; \\ 2. 数控装备可靠性教育部重点实验室 长春 130022; \\ 3. 吉林大学机械与航空航天工程学院 长春 130022)
}

\begin{abstract}
摘要: 制造系统可靠性的研究已有几十年的历史, 但相关研究所界定的研究范畴模糊, 缺乏统一的分析框架, 容易与传统的 设备系统可靠性研究发生混淆。在文献研究的基础上, 对制造系统的定义和内涵进行分析, 并据此重新阐释制造系统性能与 可靠性概念之间的区别和联系。从设备-工艺孪生的角度重新定义制造系统的概念, 并指出在制造系统可靠性研究中忽视系统 中人的作用的制造系统描述是不完备的。基于功能视角, 重新定义了制造系统失效模式, 建立制造系统可靠性分析的框架, 阐述制造系统可靠性随时间变化机制的研究方法。研究指出大数据方法将是未来制造系统可靠性研究的重要方法之一。 关键词: 文献研究; 制造系统; 可靠性; 框架模型；功能视角
\end{abstract}

中图分类号: TG156

\section{Framework and Dynamic Mechanism of Reliability Analysis of Manufacturing System}

\author{
KONG Fansen ${ }^{1,2,3}$ \\ (1. Key Laboratory of Designing and Planning for the Digital Twin Intelligent Factory, Changchun 130022; \\ 2. Key Laboratory of CNC Equipment Reliability, Ministry of Education, Changchun 130022; \\ 3. School of Mechanical and Aerospace Engineering, Jilin University, Changchun 130022)
}

\begin{abstract}
The research of manufacturing system reliability has a history of several decades, but the research scope defined by related research is fuzzy, lack of a unified analysis framework, and easy to be confused with the traditional equipment system reliability research. On the basis of literature research, the definition and connotation of manufacturing system are analyze d, and the differences and relations between the performance and reliability of manufacturing system are re explained. The concept of manufacturing system is redefined from the perspective of equipment process twinning, and it is pointed out that the description of manufacturing system neglecting the role of human in the system is incomplete, especially in the study of manufacturing system reliability. Based on the functional perspective, the failure mode of manufacturing system is redefined, the framework of manufacturing system reliability analysis is established, and the research method of the mechanism of manufacturing system reliability changing with time is discussed. It is pointed out that the big data method will be one of the important methods for reliability research of manufacturing system in the future.
\end{abstract}

Key words: literature review; manufacturing system; reliability model; framework; dynamic mechanism

\section{0 前言}

可靠性工程作为一门独立的学科已有近半 个世纪的历史, 但是, 通常所说的可靠性工程主

* 国家科技重大专项 (2017ZX04017001) 和吉林省科技发展计划 (2017010117JC，20190901009JC)资助项目。20190925 收到初稿, 20200505 收到修改稿
要是针对产品的可靠性分析、评价和优化, 目的 是评估或优化产品可靠性。而所谓系统可靠性通 常是将复杂产品看成是由若干部件或子系统所 构成的系统, 据此开展的研究, 称之为系统可靠 性研究 ${ }^{[1]}$ 。

由于制造系统定义具有广泛的外延, 因此关于 制造系统可靠性的研究国内外尚无统一的框架模型 供参考, 不同的研究人员由于其对制造系统的研究 
的视角不一样, 而采用的方法论也不一样的。本文 拟通过文献研究给出制造系统可靠性研究的统一框 架模型。为此我们需要阐释一下制造系统的内涵和 外延以及它的分类。

从系统的角度来看, 生产系统的 “实体” 转换 模式就是制造系统, 制造系统是生产系统的典型代 表; 生产系统包括制造系统(图 1)。制造系统对应于 制造企业而言; 生产系统则相对应于所有企业, 包 括制造业和服务业。因此, 生产系统在使用范围上 比制造系统大。制造过程发生于生产系统中。制造 系统的本质是制造过程的实现。

制造可分 “大制造和小制造”, “小制造” 是指 传统的 “机械制造”, 重点是指加工和装配。“大制 造” 是指产品生命周期中, 从供应市场到需求市场 整个供需链中的所有活动。前述定义显然是关于“大 制造” 系统的定义。

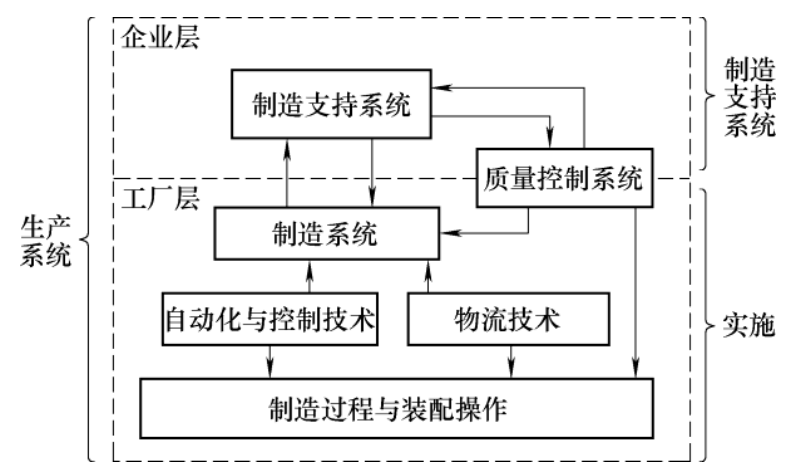

图 1 生产系统与制造系统的关系

“小制造” 系统可以被视为一组工作站, 在这些 工作站上将输入材料转换为可以销售的成品。因此 可将 “小制造” 系统定义为一个由人和设备资源所 组成的系统, 其功能是对原材料、零件或一组零件 执行一个或多个加工和/或装配操作。设备资源包 括: 生产设施、物料加工设备和工具, 物料传送装 置(夹具、工位器具)、量检具和计算机控制系统等 各种硬件; 人员是指全时或周期性巡回维持系统运 转的工作人员。

根据制造系统定义的内涵, 机械加工/装配系 统是一种典型的 “小制造” 系统。而其外延可至 一个正在制造产品的机床、加工中心、生产线(柔 性制造生产线、计算机集成制造生产线)、车间乃 至整个工厂都可看作是不同层次的制造系统 (图 2)。而关于制造系统可靠性的研究大多集中在 “小制造”，是基于 “小制造” 的制造系统可靠性 的研究。关于制造系统可靠性研究的文献出现在 上述所有层次, 侧重点各异, 下面针对各个层次 国内外研究现状简述如下。

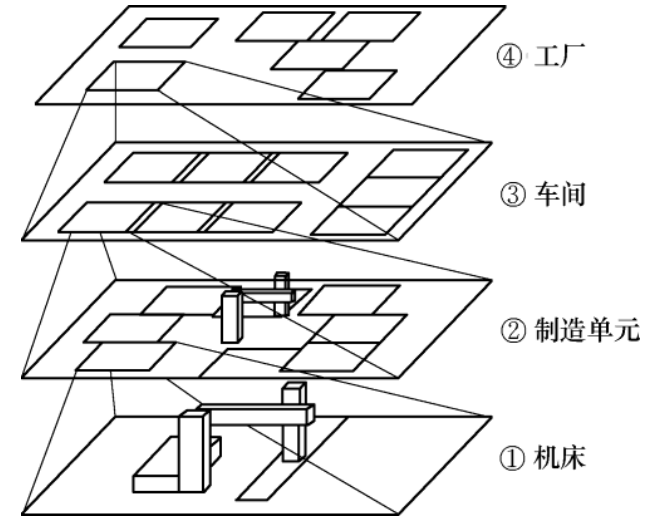

图 2 不同层次上的制造系统

\section{1 制造系统可靠性研究现状}

\section{1 国外研究}

针对离散制造系统的可靠性研究, 也可追溯到 20 世纪 70 年代初, 苏联机床研究机构——金属切 削机床科学实验研究院率先开展数控机床的制造和 试验、故障数据的统计分析等可靠性设计、试验方 面的研究 ${ }^{[2-4]}$ 。同一时期, 英国布拉德福德大学的 KELLER 等 ${ }^{[5}$ 耗时 3 年研究了约 35 台制造系统加工 单元的现场故障数据, 给出了故障间隔时间和维修 时间的对数正态分布和 Weibull 分布模型。GUPTA 等 ${ }^{[6]}$ 基于时间序列分析方法给出了数控机床的维修 策略, ARTSR 等 ${ }^{[7]}$ 研究了多种故障模式条件下混合 寿命分布参数估计的问题。日本学者研究和分析了 数控加工单元的故障模式和原因, 提出了提高数控 加工单元可靠性的措施 ${ }^{[8]}$ 。此外, FREIHEIT 等 ${ }^{[9-10]}$ 分别对影响制造系统加工单元可靠性的因素进行了 研究。这些研究主要集中在第一层次, 即围绕数控 机床以及加工中心的可靠性问题展开的。

20 世纪 70 年代初, KHODABANDEHLOO 等 ${ }^{[11-13]}$ 将可靠性研究工作引入柔性制造系统(Flexible manufacturing system, FMS)等系统级的制造装备领 域, 系统地开展了 FMS 可靠性建模与分析方法研究, 故障树分析、故障模式及影响分析、过程生成图模型 以及可靠性框图模型等。KHODABANDEHLOO 指出 在柔性制造系统规划和开发阶段的可靠性分析至关 重要, 因为它可以改变系统设计和操作理念, 提升 整个系统的性能，他引入 “制造能力”, “生产效率” 和 “交货提前期成功率” 这些指标评估柔性制造系统 的性能, 使用预测数据分析典型的柔性制造单元的可 靠性, 并讨论了设备冗余, 缓冲区库存大小和生产计 划与排程规则对系统性能的影响。CHAKRABORTY 等 ${ }^{[14]}$ 将制造系统可靠性定义为 “零件的生产订单在 
给定日期内完工的概率”。MIRIYALA 指出柔性制 造系统可靠性可以理解为 “系统能够完成所有给定 需求的稳态概率”。WANG 等 ${ }^{[15]}$ 建立了 FMS 可靠性 估计的定性模型, 并建立了与系统初始可靠性、设 备的状态、生产环境、工作经验以及维修策略等五 个方面直接相关的故障模型, 作者使用模糊信息理 论建立了柔性制造系统的可变失误率模型, 并分类 讨论了 3 种不同系统状态下失误模型的区别。HAN 等 ${ }^{[16]}$ 认为随着系统复杂性的增加, 一些可靠性分析 模式难以描述实际情况, 作者使用模糊故障树方法 描述了系统可靠性分析的模糊不确定性问题。 SAVSAR 等 ${ }^{[17]}$ 对柔性制造系统在不同运行条件下 (包括机器故障和维修) 的性能进行评价, 建立了系 统可靠性分析的马尔科夫过程模型。这些研究集中 在第二层次, 即制造单元以及柔性制造系统可靠性 的研究。

BRALL 等 ${ }^{[18]}$ 给出了工艺系统平均故障间隔时 间、工艺系统可用度等指标的计算方法。 GÖRKEMLI 等 ${ }^{[19]}$ 在 BRALL 研究的基础提出了制造 系统模糊贝叶斯可靠性与可用性评价方法。这两篇 研究是针对工艺系统的研究, 所给出的制造系统可 靠性测度方法是基于可靠性框图的。

LIN 等 ${ }^{[20-21]}$ 通过随机流网络描述了供货商与客 户之间的关系, 在给定各商品需求的前提下, 利用 代数的方法求解系统满足客户需求的概率, 并将这 一概率定义为系统的可靠性。LI 等 ${ }^{[22]}$ 探讨建立了在 有限缓存区和机器不可靠的情况下, 基于交期表现 的客户满意度模型, 并通过迭代的方法对模型进行 求解。SAVSAR 等 ${ }^{[23]}$ 建立了在制品恒定的情况下串 行生产线的可靠性模型, 并运用离散事件仿真的方 法验证了该模型。AN 等 ${ }^{[24]}$ 重点描述了制造过程的 可靠性指标与系统输入参数之间的关系, 并利用线 性代数的方法对实际收集到的数据进行拟合建模。

GARG 等 ${ }^{[25]}$ 使用模糊非线性规划研究了串联制造系 统的多目标可靠性冗余分配问题, 作者所提出的模 型具有柔性, 并适于人的决策过程。WU 等 ${ }^{[26]}$ 在考 虑机器可靠性基础上, 以实现生产线产能的最大化 为目标, 研究了串行生产线可重构资源的分配问题。 CONWAY 等 ${ }^{[27]}$ 在考虑有无缓冲区和机器可靠性的 条件下, 研究了串联制造系统的在制品库存问题, 研究表明缓冲区的位置和容量对在制品库存和系统 产出率具有显著影响。HANDFIELD等 ${ }^{[28]}$ 根据对 193 家制造企业的调查发现生产计划、库存和生产调度 能力对交货可靠性有显著影响。 EMREENGINARLAR 等 ${ }^{[29]}$ 的研究指出: 当机器故
障服从指数分布、埃尔朗分布和瑞利分布时, 系统 所需的最小缓冲区容量与机器的工作时间无关，与 机器的可用率成反比。KOREN 等 ${ }^{[30]}$ 研究了 6 种串 并联生产组织模式对系统性能的影响, 并从可靠性、 质量、生产周期和成本四个方面对生产系统的性能 进行了对比分析。在制造过程可靠性研究领域, $\mathrm{SHI}^{[31]}$ 提出的多阶段制造的误差流分析理论和方法 是解决制造过程可靠性的重要建模方法, 他通过对 基于灵敏度设计评价、基于过程的误差综合、最优 传感器分布策略研究、可诊断性研究、故障诊断以 及质量可靠性链的研究建立了一个多阶段制造误差 递减分析的统一框架。这部分研究主要集中在串联 生产线并以满足客户需求为目标的可靠性研究是第 三和第四层次的研究，所给出的制造系统可靠性测 度方法是基于马尔可夫链的。

\section{2 国内研究}

国内研究主要分三类, 第一类是第一层面也就 是机器或加工中心层面的可靠性研究; 第二类是关 于制造系统层面可靠性的研究; 第三类是关于制造 过程工艺可靠性问题的研究。国家中长期科学和技 术发展规划纲要(2006-2020)确定了 16 个国家科技 重大专项, 专项中所涉及的数控装备可靠性问题主 要是针对数控机床以及加工中心整机与关键领部件 可靠性的研究, 也就是第一类研究。其中具有代表 性的研究主要有吉林大学数控装备可靠性教育部重 点实验室关于可靠性试验技术的研究、重庆大学可 靠性研究团队关于质量与可靠性的研究、南京理工 大学可靠性研究团队关于滚动功能部件的可靠性研 究、东北大学可靠性研究团队关于可靠性设计领域 的研究、电子科技大学可靠性研究团队关于可靠性 建模与评价的研究等。第二类是关于制造系统层面 可靠性的研究主要有我国在“八五”期间, 对柔性制 造系统可靠性进行了系统全面地理论分析, 并在北 京 FMS 中心与长春 FMS 中心进行了系统的应用研 究 ${ }^{[32]}$, 其中胡华平 ${ }^{[33]}$ 提出了 FMS 多任务可靠性建 模方法。

陈艳霞 ${ }^{[34]}$ 提出了广义随机 Petri 网和动态故障 树相结合的 FMS 可靠性分析模型; 高振清等 ${ }^{[35]}$ 设 计了一种车间制造系统控制模型, 该模型可通过系 统分析直接建立 GO 图, 并运用状态组合算法依据 该模型进行车间制造系统可靠性分析; 周丰旭等 ${ }^{[36]}$ 建立一种基于状态熵的多态制造系统可靠性分析模 型, 作者采用从元件到子系统、从子系统到系统的 层级划分方法对多态制造系统进行性能分析, 结合 实例验证了所建模型的合理性。杨林 ${ }^{[37]}$ 以串行制造 
系统为研究对象, 重新定义了制造系统的可靠性和 失效性, 作者将人员、信息、组织、环境、物料、 设备等因素纳入到系统可靠性研究范畴, 不再单纯 局限于由设备或者人员引起的生产故障。

总的来说, 制造系统的早期可靠性研究工作主 要是以设备故障为中心, 采用的是单元级的可靠性 分析方法, 而这些静态可靠性模型与分析方法并不 能很好表达制造系统运行过程中各设备单元间极其 错综复杂的关系, 缺乏描述系统运行过程、状态传 递与故障演变等特性的能力, 难以满足复杂制造系 统运行可靠性分析的需要。

从制造系统运行可靠性角度开展的研究主要有 柳剑 ${ }^{[38]}$ 利用脆性理论和多状态系统理论研究制造 系统的运行可靠性和维修保障策略, 探讨了制造系 统脆性效应的累积与传播机理, 建立了基于广义外 在环境可靠性分析“ $R-E$ ”体系的制造系统运行可靠 性分析框架与性能状态演变预测模型, 提出了考虑 脆性效应的改进区间通用生成函数可靠性评估方 法。李娟 ${ }^{[39]}$ 对制造过程中交错复杂的质量与可靠性 等相关信息加以辨识, 在分析产品质量与制造系统 可靠性之间关联关系的基础上，从“制造系统-制造 过程-产品”三个维度拓展延伸了 $\mathrm{QR}$ 链。胡毓婷 ${ }^{[40]}$ 结合生产实际对变速箱壳体生产线的可靠性进行了 横向与纵向对比研究, 采用模糊综合评价法对两条 壳体生产线进行多个可靠性指标的横向比较, 通过 现场考察和信息收集, 分析其可靠性产生差异的原 因, 并提出改善建议; 针对同一生产线采用灰色多 层次综合评判模型, 沿时间轴方向, 观察壳体生产 线各时间段可靠性随时间变化的趋势。通过理论与 实际的结合, 分析其可靠性变化的原因, 并提出可 行的改善措施。从中蔚 ${ }^{[41]}$ 对生产系统可靠性及失效 等相关问题进行描述, 并在生产系统可靠性预计的 基础上, 提出了适用于生产系统可靠性分配的方法。 张丁等 ${ }^{[42]}$ 提出了一种新的基于故障损失的有效系 统可靠性评估方法, 并以故障损失为基础, 将零部 件重要性测度用于设备的重要性分析。前者反映了 系统的可靠性现状, 后者指出了提高可靠性的途径。 作者采用动态有向网络对制造系统的可靠性进行描 述和建模。

第三类研究是关于工艺可靠性的研究, 本文作 者认为这类研究是介于第一和第二类研究之间的研 究。工艺可靠性的研究目标是通过综合考虑材料、 设备、人员等过程特性的波动规律, 以最终产品的 质量为优化目标, 对多个制造活动进行统筹规划, 提高在规定的工艺条件下和规定的生产周期内, 所
加工的产品达到规定质量和可靠性要求的能力。有 关这一领域的研究主要有匡芬 ${ }^{[43-44]}$ 为定量评估加工 过程中各种随机因素对加工过程可靠性的影响, 从 完成生产任务的角度出发, 将生产任务的时间(T)、 质量 $(\mathrm{Q})$ 、成本 $(\mathrm{C})$ 均看作产品特性, 研究了加工过 程可靠度的概念, 分析了加工工艺与产品质量特性 的关联机理, 参考广义应力一强度干涉理论, 提出 了加工过程的任务载荷一生产能力模型, 建立了加 工过程可靠性状态函数, 并给出了评估框架。付桂 翠等 ${ }^{[45]}$ 以提高产品的可靠性为目的, 分析了工艺系 统、工艺过程产品输出参数、产品使用性能、产品 可靠性四者的关系。在此基础上, 提出了基于产品 可靠性的工艺系统基本影响关系可靠性模型。上海 交通大学徐锐等 ${ }^{[46]}$ 利用马尔科夫模型, 根据合并迭 代的思想建立了关联多工序制造系统的加工质量分 析模型, 提出了系统质量瓶颈加工工序的判定方法, 从系统参数的角度提出了改进加工质量的建议。宋 婷婷 ${ }^{[47]}$ 则以关联多工序装配系统为研究对象, 考虑 上游工序对下游工序的质量影响, 运用马尔科夫建 模思想, 建立了多工序装配系统的马尔科夫模型, 提出了装配系统中瓶颈工序的判定方法。邓超等 ${ }^{[88]}$ 提出一种基于切削动力学模型的工艺可靠性评估方 法, 作者通过评价加工精度的达标概率来评价设备 工艺可靠性。张根保等 ${ }^{[49]}$ 并利用灰色预测理论建立 了装配过程可靠性与关键可靠性控制点的相关关 系, 以此来评价和预测装配过程的可靠性。CHEN 等 ${ }^{[50-51]}$ 用 Q-R 链刻画制造系统的产品质量流转及其 与设备可靠性的交叉影响, 以评价制造过程的可靠 性。吴军等 ${ }^{[52]}$ 从数控装备加工过程动力学建模的角 度研究了工艺可靠性仿真方法, 他们首先建立了数 控装备铣削加工过程的非线性动力学模型, 以分析 不同工艺条件下数控装备铣削加工性能, 在此基础 上采用支持向量回归机建立了数控装备铣削加工性 能映射模型及其工艺可靠性仿真模型。杜彦彬 ${ }^{[53]}$ 依 据再制造工艺过程对机床可靠性的影响, 提出机床 再制造工艺过程可靠性的概念; 在分析机床再制造 工艺过程可靠性影响因素的基础上，建立了机床再 制造工艺过程可靠性 GO 图模型及其概率模型。

JIANG 等 ${ }^{[54]}$ 使用制造系统可靠性分析的 Petri 网模 型描述了工艺系统的同步、资源共享、冲突等现象。 MACIEJ 等 ${ }^{[55]}$ 对生产过程可靠性评价方法进行了详 细综述, 在文献研究基础上, 作者基于生产过程可 靠性所涉及的五个方面(机器和设备的性能及其发 生故障的可能性、维护和物流支持基础设施的性能 及其发生故障的可能性、信息流和信息可靠性、发 
生不希望发生的随机危害/威胁的可能性(内部和外 部类型)、决策者决策的过程和人为因素的可靠性) 建立一个多维生产过程可靠性综合评价流程框架。

从以上文献可知, 各国学者对产品制造过程、 制造设备以及离散制造系统可靠性概念的定义大概 分为三类: 一类是基于传统可靠性定义的, 即制造 系统在时刻 $t$ 正常工作的稳态概率; 另一类是从制 造系统的功能出发, 即制造系统满足客户需求或相 应系统功能的能力。最后一类是工艺可靠性问题, 这是一个技术可靠性的问题, 无法使用传统方法进 行定义。综上所述, 国内外学者对制造系统可靠性 的研究虽然已有几十年的历史, 但这些研究所界定 的研究范畴模糊, 缺乏统一的分析框架, 容易与传 统的(产品)可靠性分析或者设备(机床)可靠性研究 发生混淆。本文将在文献研究的基础上, 对制造系 统的定义、内涵及其主要构成要素进行分析, 在讨 论制造系统性能的同时讨论其与传统可靠性概念之 间的区别和联系, 对制造系统功能进行分析, 最终 结合自己多年在制造系统理论研究中的认识建立制 造系统可靠性分析统一框架, 为制造系统可靠性的 研究提供一种新的思路。

\section{2 制造系统的故障与可靠性分析}

为了准确定义制造系统可靠性的内涵, 本文作 者定义制造系统概念: 制造系统是由设备资源、人、 料、环境与安全、及工艺子系统组成的系统, 即由 设备资源子系统和工艺过程子系统构成的设备一工 艺孪生系统, 其功能是对原材料、零件或一组零件 执行一个或多个加工和/或装配操作。设备资源包 括: 生产设施、物料加工设备和工具, 物料传送装 置(夹具、工位器具)、量检具和计算机控制系统等 各种硬件; 人员是指全时或周期性巡回维持系统运 转的工作人员; 料是指原材料; 环境与安全是指工 作环境及其安全状态; 工艺子系统包括工艺方法和 工艺参数。

本文定义的制造系统的概念与前述文献中定义 的 “小制造系统” 和 “大制造系统” 的概念都不同, 近于 “小制造系统” 的概念。因为没有设备的工艺 系统是虚拟的生产系统, 而离开工艺系统的设备系 统是不具有生命力的系统, 在相同的设备系统中, 使用不同工艺方法和工艺参数会有不同产量和质量 的产品产出, 因此, 本文作者将制造系统视为由设 备资源子系统和工艺过程子系统构成的设备一工艺 孪生系统。并基于这一定义给出制造系统故障模式 和可靠性分析框架模型。

\section{1 制造系统的故障与失效模式分析}

1978 年, HIMMELBLAU ${ }^{[56]}$ 在他出版的故障诊 断方面的第一部学术专著里最早给出了故障的定 义, 即系统至少有一个可观测或可计算的重要变量 或特性偏离了正常范围。

对于运行中的制造系统, 本文将其故障分为设 备系统故障、性能退化以及工艺系统故障。

(1) 设备系统故障。设备系统故障主要表现为 两类, 即功能性故障与非功能性故障。功能性故障 的主要表现为参数问题, 如输出的工件质量不满足 技术规范; 功能问题, 如元器件或零部件与系统之 间的位置、运行关系不正常。而非功能性故障的一 般表现形式有轻微振动、异响、漏油等。

(2) 设备系统性能退化。制造系统的状态性能 具有连续退化属性, 其整体的性能状态是由各组 成系统要素的性能状态决定的, 是系统要素相互 作用的结果。在实际加工中通常由于磨损、松动、 使用等原因，使得设备状态呈现衰退老化甚至失 效等状况, 且随着使用时间和维修次数的累积增 加, 系统整体性能水平的衰退速度会逐渐加快, 使得其性能呈现不同状态。此外, 由于制造任务 的复杂多变性、制造环境的动态不确定性、技术 水平不等的操作员等都将导致系统性能退化速度 超过设计预期。

(3) 工艺系统故障。工艺系统失效模式在文 献[52]中给出一个定义, 作者定义工艺过程故障为 所有除设备故障以外因素导致的生产线停线和不合 格品生产的生产事件。这一故障模式不存在退化问 题。上述关于制造系统的故障定义是基于第一层面 的制造系统, 即从机床、加工中心等机器的角度定 义的故障，而本文关注的是从第二、三、四层面如 何定义制造系统的故障、失效模式及其分析方法。 本文将生产单元中存在的人机干涉视为一种故障状 态, 就以设备为中心的生产系统而言, 分两种情况: 一是一人一机生产系统, 忽视人的作用, 按前述定 义设备系统与工艺系统故障; 二是一人多机生产单 元, 当发生机器等人现象时, 即发生人机干涉, 本 文将其视为一种故障。就以人为中心的生产系统而 言, 如手工装配系统, 当多人共用一台设备/工具, 出现人等设备/工具状态时, 将其视为一种故障状 态。此外, 就考虑人的作用的系统, 人误也将被视 为一种故障形式, 详见文献[57]。

\section{2 制造系统失效模式分析模型框架}

本文作者认为制造系统是由若干设备所组成的 物理系统和由若干工序所组成的虚拟系统在人的协 同下在规定时间内、按照规定的成本完成规定生产 
任务的系统。因此，作者认为制造系统的失效模式结果，基于这种认识作者给出如表 1 所示制造系统 是由设备资源物理系统、人与工艺系统耦合作用的＼cjkstart失效模式分析框架。

表 1 制造系统失效模式分析框架

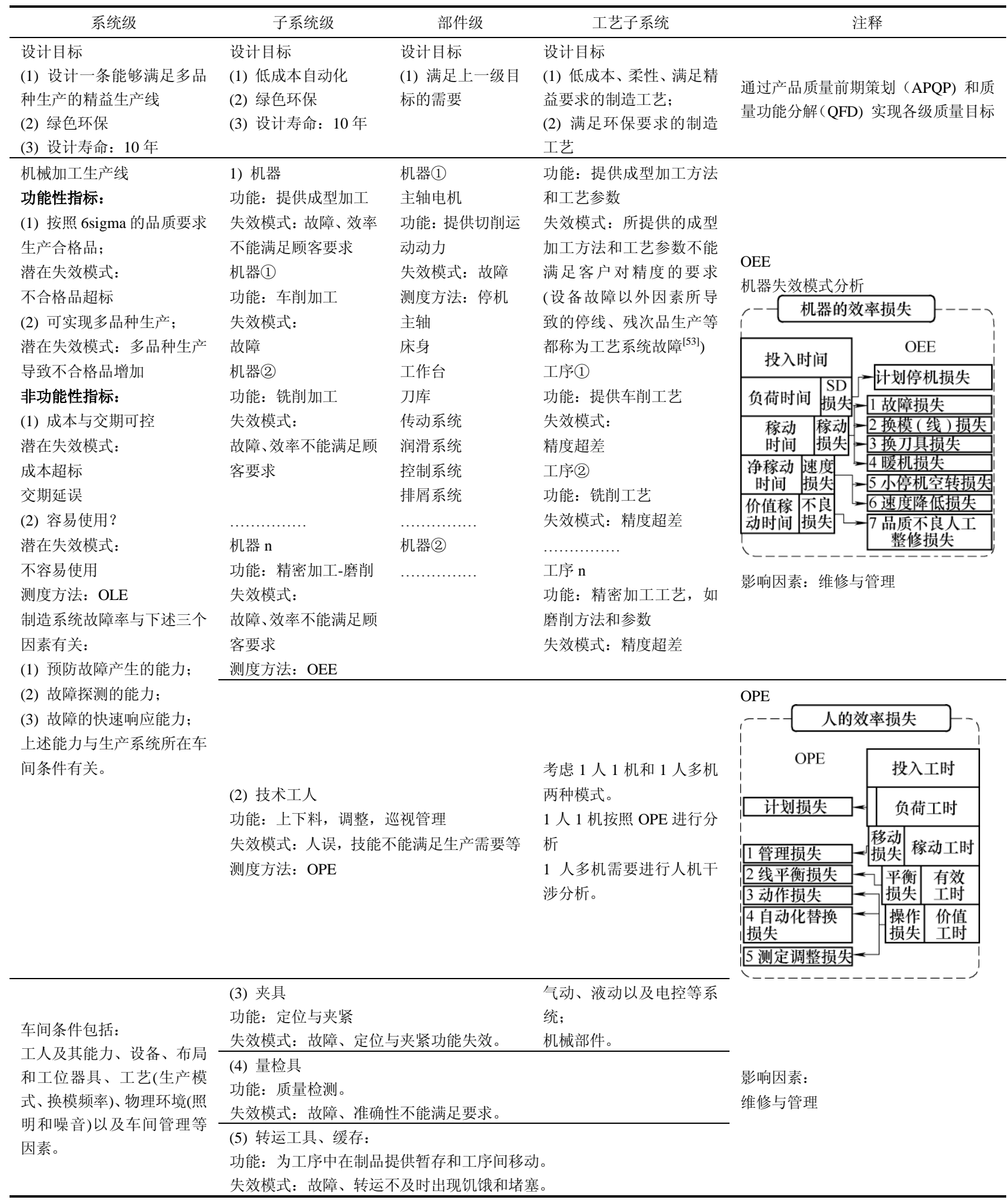

依据该框架可从五个方面探讨制造系统的失效 问题: (1) 从设备本身的综合效率角度研究故障形 式; (2) 从人的角度研究效率和质量损失; (3) 从工 艺系统角度研究效率和质量损失; (4) 从设备-工艺
匹配度上研究效率和质量损失; (5) 综合上述四个方 面, 从复杂性角度研究效率和质量损失。

文献[56]使用采用瞬时合格产品生产率(Instantaneous production rate，IPR)描述制造系统及其设备 
单元的性能状态, 它表示单位时间内合格产品的产 量, 蕴含了对制造系统功能可靠性与参数可靠性的 表征。制造系统具有若干个离散状态分别对应各自 的 IPR 值, 当制造系统性能状态退化导致无法满足 生产需求时, 制造系统可视为发生故障。IPR 值作 为制造系统最终功能输出的性能特征参数, 它所表 征的制造系统故障是一种广义的故障, 既包含了所 有的功能性故障与非功能性故障, 又包含了所有的 参数性故障, IPR 值正是对这种广义故障形成、发 展与发生的统一描述。而狭义故障是由载荷、故障 机制与故障模式三要素共同导致的具体故障, 是广 义故障最终形成的具体根源。按照制造系统的物理 特性分, 其具体故障可以分为机械故障、电气故障 和信息故障; 按照制造系统的单元组成分, 其具体 故障可以分为加工单元故障、物料储运单元故障、 刀具系统故障、质量检测系统故障和中央控制系统 故障; 按照制造系统的故障起因分, 其具体故障又 可以分为认知故障、设备故障、设计故障、制造故 障、元器件故障和干扰故障等。

本文考虑的制造系统的故障与上述广义故障形 式相似, 即将制造系统性能无法满足生产需求的情 况视为制造系统的故障, 但是与狭义故障略有不同, 既包括由载荷、故障机制与故障模式三要素共同决 定的具体故障, 也包括人误、工艺参数漂移或设备、 工艺方法和工艺参数匹配不合理导致的故障。同时 作者认为制造系统性能退化可通过调整工艺方法和 工艺参数予以补偿。

\section{3 制造系统可靠性的定义}

在 GB 318-1982 中对可靠性作了如下定义: 产品在规定的条件下和规定的时间区间内完成规定 功能的能力。

可靠性有狭义与广义之分, 狭义的可靠性仅指 产品在规定条件下和规定时间内完成规定功能的能 力, 主要针对不可修产品而言的; 而广义的可靠性 通常还包含维修性等方面的内容, 更多的是针对可 修产品而言。

制造系统这类系统即是可修的、也是可调整性 的产品, 所谓的可调整性是指在其设备系统功能退 化时, 可适当采用工艺系统(方法或参数) 补偿的方 法来达到生产合格品的目的。

按 GB 318-1982 可将制造系统可靠性定义为 制造系统在规定的条件下和规定的时间区间内完成 规定功能的能力。

这里规定的条件是指系统的运行条件, 如温 度、湿度、灰尘、振动等环境条件, 以及加工范 围、加工材料、规定的维修保养、企业管理制度
等运行条件等。规定的时间是指系统设计时所确 定的服役寿命(包括班次和每班的工作时间), 如 可以考查系统在一个交期内的可靠性, 也可以考 查系统在整个服役阶段的可靠性(包括维修后系 统的可靠性)。规定的功能是指系统应该完成的制 造任务, 如工件的自动上下料、加工的自动工作 循环、自动换刀、工件的自动存储和输送、自动 排屑、加工精度的自动检测以及工件加工精度、 生产率的保证等。

上述关于可靠性的定义与本文所定义的制造系 统的设备资源系统相关，但是没有考虑制造系统的 另外两个要素即人和工艺系统。

谈可靠性一般与故障相关, 如前述, 本文定义 的制造系统故障可分为两类: 狭义故障、人误、工 艺参数漂移或设备、工艺方法和工艺参数匹配不合 理等导致的设备系统故障和工艺系统故障。本文定 义的设备系统故障即为前述狭义故障, 其余如工艺 参数漂移或设备、工艺方法和工艺参数匹配不合理 等为工艺系统故障。

制造企业在进行产品制造时必须同时关注设备 系统故障和工艺系统故障, 用户对制造系统可靠性 最关心的问题就是降低其运行过程中的故障率, 减 少人误、设备系统故障和工艺系统故障的发生, 使 制造系统处于“随时可用”的状态。

基于本文所给出的制造系统的定义和上述对制 造系统故障模式的诠释，作者给出了如图 3 所示制 造系统可靠性分析的框架和动力学机制。

由于制造系统整体工作性能状态、设备系统的 退化程度、工艺系统与设备系统的匹配程度, 反映 在输出终端就是可执行部件的动作异常变化, 这将 直接导致加工对象的数量和精度不符合要求(即不 能完成规定的任务), 最终造成生产能力的下降。因 此根据制造系统最终功能的宏观输出, 本文作者从 使用者的视角依据制造系统的输出评价指标给出其 一般性的定义, 即我们将制造系统可靠性定义为在 规定的工艺和使用条件下, 按照规定的成本, 在规 定的时间内(交期)生产规定数量合格品的概率。这 一定义涵盖了图 3 所示制造系统输出性能的四大评 价指标, 即产成品的数量、质量、交期和成本。它 表示制造系统在单位时间内生产合格产品的数量满 足需求的概率, 蕴含了对制造系统中设备子系统可 靠性与工艺子系统可靠性的表征。下面给出传统(产 品)可靠性与制造系统可靠性定义在几个关键要素 上的区别, 见表 2 。

由定义可知, 本文考虑的制造系统功能是制造 能力, 制造质量和交货周期等。 


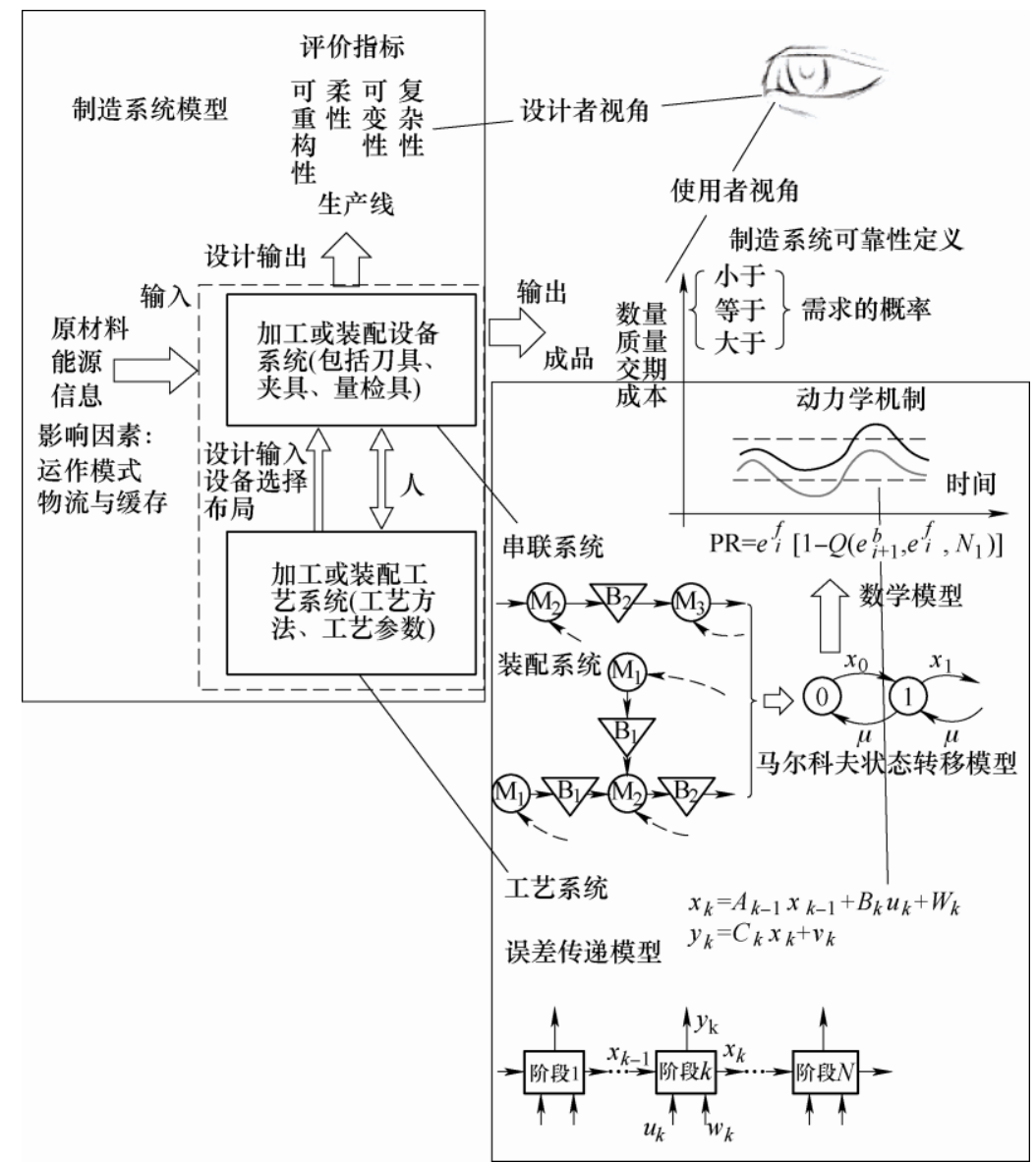

图 3 制造系统可靠性分析的框架、模型和动力学机制

表 2 设备可靠性、工艺系统可靠性与制造系统运行可靠性分析对比

\begin{tabular}{|c|c|c|c|}
\hline & 设备可靠性 & 工艺系统可靠性 & 制造系统运行可靠性 \\
\hline 时间 & 设备使用时间/寿命周期 & 与时间没有直接关系 & 生产周期/提前期 \\
\hline 条件 & $\begin{array}{l}\text { 设备使用环境和载荷符合 } \\
\text { 要求等 }\end{array}$ & 工艺装备使用环境和载荷符合要求等 & $\begin{array}{l}\text { 在制造系统使用环境与任务未发生重大变化的 } \\
\text { 情境下 }\end{array}$ \\
\hline 功能 & $\begin{array}{l}\text { 从用户角度进行的设备功 } \\
\text { 能定义 }\end{array}$ & $\begin{array}{l}\text { 从工艺过程的角度进行功能的定义(提 } \\
\text { 供工艺方法和工艺参数) }\end{array}$ & $\begin{array}{l}\text { 主要从制造系统本身出发, 同时考虑外部需求和 } \\
\text { 企业运营者的战略着眼点 }\end{array}$ \\
\hline 故障 & $\begin{array}{l}\text { 从用户角度进行的设备故 } \\
\text { 障或是否满足性能要求来定 } \\
\text { 义 } \\
\text { 系统内零部件故障之间相 } \\
\text { 关, 一个元素的故障可导致另 } \\
\text { 一个元素的故障, 组成系统的 } \\
\text { 元素之间有串并联之分, 可用 } \\
\text { 串并联可靠性框图进行分析 }\end{array}$ & $\begin{array}{l}\text { 所提供的非设备工艺要素不能满足客 } \\
\text { 户对产品精度的要求 }\end{array}$ & $\begin{array}{l}\text { 从制造系统本身出发定义影响制造系统功能实 } \\
\text { 现的故障 } \\
\text { 系统中各个设备故障由于缓存区作用彼此相关 } \\
\text { 性不大, 一个制造单元的故障对另一制造单元的影 } \\
\text { 响有限, 各个制造单元具有串联关系或装配关系。 } \\
\text { 仅从设备角度讨论制造系统故障是不全面的, 需要 } \\
\text { 考虑非设备故障引起的停机和残次品故障 }\end{array}$ \\
\hline 主要 & $\begin{array}{l}\text { 是设备质量在使用时间上 } \\
\text { 的体现 }\end{array}$ & $\begin{array}{l}\text { 是工艺系统可靠性在制造过程中的体 } \\
\text { 现 }\end{array}$ & $\begin{array}{l}\text { 从系统的角度了解和评估制造系统的各项能力, } \\
\text { 是制造系统性能在时间上的体现 }\end{array}$ \\
\hline
\end{tabular}

\section{3 制造系统可靠性模型及动力学机制}

\section{1 设备系统模型}

模型是指产生某种现象的结构, 包括构成要素 及其相互关系，本文从制造系统运行和设计的角度 建立图 3 所示制造系统可靠性分析模型，从运维的
角度看制造系统的可靠性，其主要影响因素有：原 材料、能源、信息和生产线的结构可靠性会影响制 造系统的可靠性, 从设计的角度看人与设备的选择、 布局、物流与缓存以及运作模式等均会影响制造系 统的可靠性。狭义制造系统中最常见的生产组织结 构是生产线, 由一系列的机器设备或工作站组成, 机器之间可能被缓存区分离开，又或者是检查站。 
接下来对这些关键要素进行阐述。

(1) 设备/工作站。对设备来说, 存在运行与停 机两种状态。当设备处于运行状态时, 系统中的零 件还有排队和缺陷两种状态, 缺陷会导致返工; 就 机器而言存在饥饿和堵塞两种状态。当设备处于停 机时, 设备处于故障或维修状态。上述为与设备和 工作站有关的不可靠状态。

(2) 缓存区。缓存区的存在属于系统配置问题, 有些系统不设置缓存, 有些系统设置缓存或者在部 分工作站之间设置缓存, 它可以使制造系统变成柔 性的连接, 一般认为缓存的设置有利于提高制造系 统的可靠性, 但研究有缓存的制造系统存在系统状 态增多的问题。

(3) 检查站。检查站的存在也属于系统配置问 题。检查站会检验上游的一个设备或一组设备生产 的零件的质量缺陷, 当然, 检验过程也不是完全可 靠的, 会出现错误而导致被检零件归入错误的分 类。确定产品是否报废或返工的工作一般由检查站 完成。

\section{2 设备系统组成要素关系模型}

设备系统可靠性模型按物理结构分串联型和并 联型, 这里我们限定进行离散制造系统可靠性研究 的系统结构为串行结构或装配结构。

串行结构生产线: 如图 4 所示, 将生产单元(方 块) 以串行方式连接起来, 并通过物料储运设备(圆 圈)将工件从一个生产单元输送到与它相邻的下一 个生产单元的生产系统。进一步分带成品库存的串 行生产线、闭环生产线、带产品质量监测的串行生 产线, 允许再加工的串行生产线以及可重入式生产 线等。这里我们将一人多机生产单元(同构任务并行 机器和异构任务串行机器) 按图 5 所示简化为串行结 构生产线进行可靠性分析, 具体方法详见文献[58]。

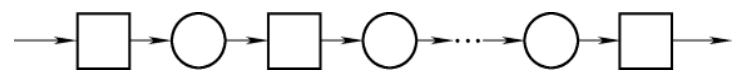

图 4 串行生产线

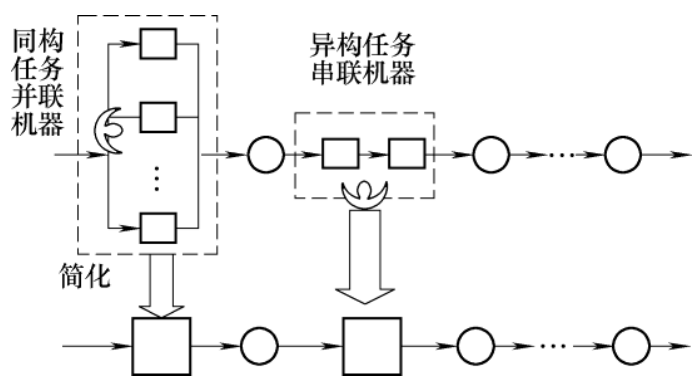

图 5 简化后的串行生产线

装配系统: 两条以上的串行生产线(部件生产线) 通过一个或几个合并操作连接将各部件组装起来,
并对组装品进行其他后续加工操作的生产系统。主 要包括单一组装操作的装配系统、含有多个组装操 作的装配系统以及复杂生产系统。现实中的制造系 统不会像前面线性系统这么简单, 它往往更复杂。 鉴于当前制造企业模块化生产和外包生产的现状， 制造系统通常由一条较短的主线和多条支线构成, 如对于一条装配线来说, 支线就是分装线。图 6 展 现了这样一个系统, 与线性系统不同, 这里原材料 从每条线的开始端进入系统。

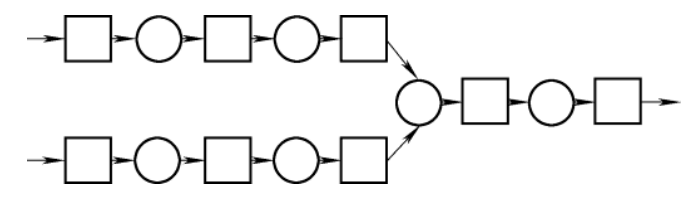

图 6 装配系统

\section{3 设备-工艺孪生系统模型}

这里我们定义工艺系统由非设备工艺要素组 成, 是一个与设备系统伴生的虚拟系统, 它由与设 备系统中各个设备相对应的工艺方法、工艺参数等 组成。工艺系统我们也可将其视为信息系统, 设备 系统相当于物流系统, 信息系统与物流系统的准确 同步才能确保制造系统的可靠运行, 如图 7 所示。

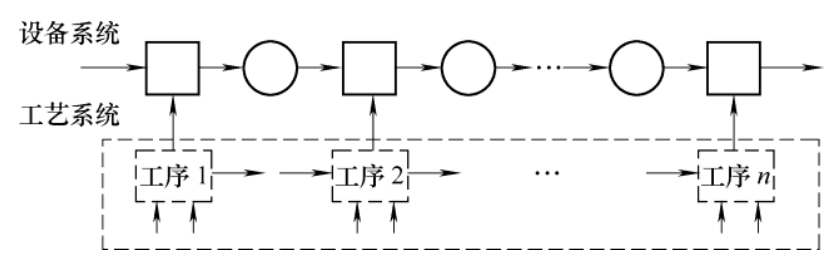

图 7 设备-工艺孪生系

\section{4 制造系统模型}

制造系统由设备系统、工艺系统以及生产支撑 系统组成, 并受人、机、料、法、环、测生产过程 六要素及其控制策略等生产要素的影响。因此, 制 造系统被定义为如图 8 所示的由设备物理系统与信 息系统共同构成的制造系统。

\section{5 由模型产生的动力学机制}

本文拟通过对制造系统可靠性模型及动力学机 制的研究来捕捉制造系统可靠性的本质, 而所谓本 质是模型与动力学机制的相互作用。所谓动力学机 制是以长远目光观察模型产生的现象, 以及今后将 会产生怎样的结果及动向, 即会出现怎样的模式。 本文将从模型及其动力学机制的角度来考察制造系 统的可靠性问题。

\subsection{1 设备系统基于迭代的方法}

关于生产线建模的方法主要有两种, 一种是数 学建模的方法, 另一种是计算机仿真的方法。其中关 于生产线数学建模的方法主要有随机流网络法 ${ }^{[20-21]}$, 


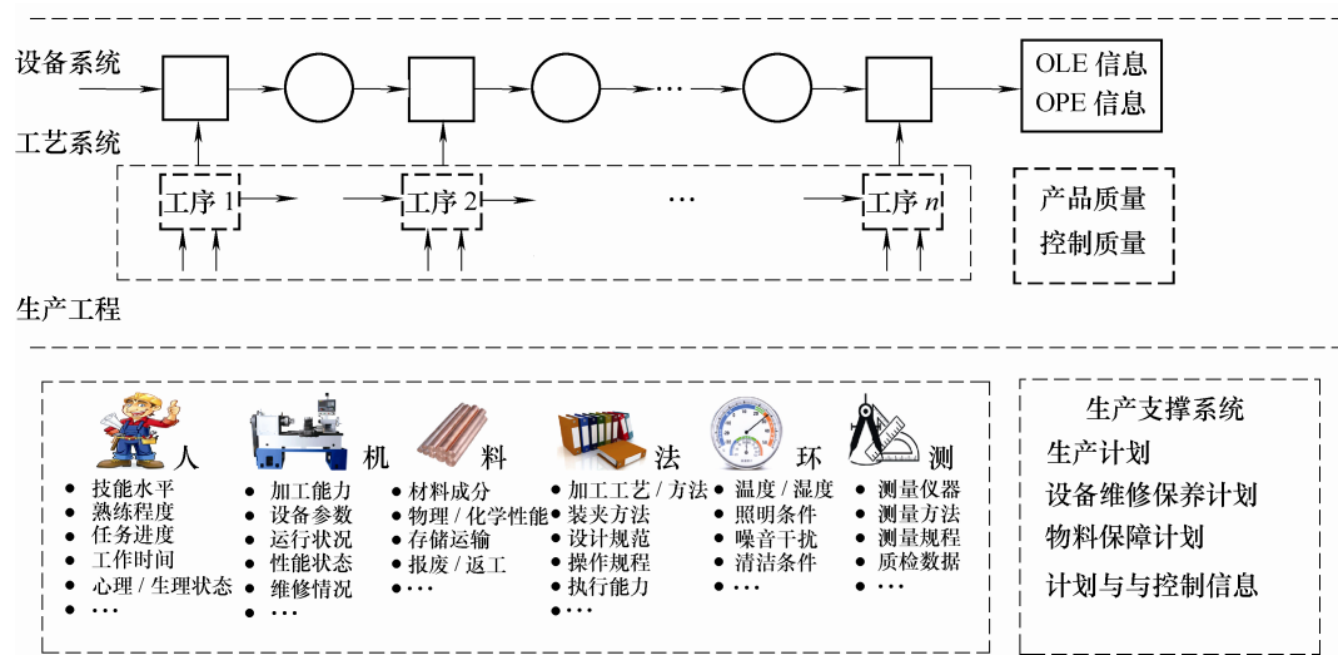

图 8 由设备物理系统与信息系统共同构成的制造系统模型

排队论方法和开闭环排队网络方法、分解法、以及 基于马尔科夫过程的合并法等 ${ }^{[59]}$ 。其中对于多机串 联生产线效率的研究, 由于不存在精确解, 目前应 用最广的两种近似求解方法分别为合并法和分解 法。LI 等 ${ }^{[60]}$ 提出了多机串联生产线数学模型求解的 合并法, 他将串联的两个机器合并为一个虚拟机器, 并假设虚拟机器与串联的两个机器具有相同的效 率, 即按效率等价的思想, 顺着串联生产线物理顺 序向前和向后依次进行合并求解系统的性能参数。 GERSHWIN ${ }^{[61]}$ 提出了串联生产线数学模型求解的 分解法, 他将多机串联生产线划分为一系列的两机 串联生产线, 针对所有的两机生产线建立参数方程, 并建立一种分解运算法则来求解这些未知数。

GERSHWIN 针对缓存区容量有限、机器有随机故障 的多机串联生产线, 提出了对其进行效率分析的分 解法, 这个近似方法的基础是基于系统特性的, 如 流守恒。通过上述方法我们得到的是制造系统的生 产率, 而不是系统的可靠性, 为了得到制造系统的 可靠性测度指标, 我们需要对它进行长期观察, 就 制造系统而言, 可直接观察到的数据是生产率, 间 接观察到是在规定时间内, 以规定的成本生产合格 品的数量满足客户需求的概率, 这是本文定义的制 造系统可靠性的概念。

\subsection{2 工艺系统基于误差流的动力学机制}

进行工艺系统可靠性动力学机制描述比较相近 的方法有两种, 一种是由美国佐治亚理工大学史建 军教授 ${ }^{[31}$ 提出的机械加工系统的误差流分析理论 和方法, 如果我们在制造系统可靠性研究中把加工 误差定义为故障, 那么误差流分析方法是从系统角 度研究制造系统故障传递机理的有效方法, 根据该 方法可以给出误差(精度失效)在各个工作站之间的 传递机理。另一种是由 $\mathrm{CHEN}^{[50-51]}$ 等提出的 Q-R
链模型, 用 Q-R 链刻画制造系统的产品质量流转及 其与设备可靠性的交叉影响, 从而评价制造系统的 可靠性。但是这两种方法均未将本文所关注的工艺 方法和参数当作显性变量进行描述。误差流方法给 出的是误差在各个工作站之间的流转机制, 而 Q-R 链刻画制造系统的产品质量流转及其与设备可靠性 的交叉影响。

\subsection{3 制造系统基于大数据分析的动力学机制}

传统上制造系统可靠性测度方法主要有可靠性 框图法和马尔科夫链方法。依据本文的定义, 制造 系统可靠性影响因素众多, 首先需要考虑的是设备 系统中各个设备的可靠性数据、故障模式以及维修 记录等; 其次要考虑各个工作站的工艺方法和工艺 参数, 例如车削方法中需要考虑背吃刀量、主轴转 速和进给速度等, 最后还要考虑生产过程中的人、 机、料、法环、测等生产过程影响因素。按前述的 定义制造系统由设备系统、工艺系统以及生产支撑 系统组成, 受人、机、料、法、环、测等生产过程 诸多要素影响, 鉴于上述原因建议使用大数据的方 法构造制造系统的可靠性动力学演化模型。

\section{4 结论与展望}

本文作者在进行文献研究时发现关于制造系统 及其可靠性的定义各国学者均从自己的研究实际, 依据自己的研究背景定义制造系统, 并据此开展相 应的可靠性研究。但是这些制造系统及其可靠性的 定义大多是基于设备系统的定义, 本文作者认为制 造系统区别于制造设备的关键在于: (1) 含有人的要 素; (2) 制造设备只有赋予相应的工艺要素, 如工艺 方法和工艺参数才可称为制造系统。为此本文从设 备-工艺孪生的角度重新定义了制造系统的概念, 并 
指出忽视系统中人及其他工艺要素作用的制造系统 描述是不完备, 基于上述认识, 讨论了制造系统失 效模式, 建立了制造系统可靠性分析的框架模型, 并据此探讨了制造系统可靠性随时间变化的动力学 机制的描述方法。研究指出大数据方法是未来制造 系统可靠性研究的重要方法之一。

\section{参 考 文 献}

[1] 包伟伟. 制造系统可靠性分析框架与评价模型研究[D]. 长春: 吉林大学, 2018.

BAO Weiwei. Research on reliability analysis framework and evaluation model of manufacturing system[D]. Changchun: Jilin University, 2018.

[2] CHIRKOV A L, MAKAROV N V, GAVRYUSHIN A I, et al. Enhancing the reliability of $\mathrm{NC}$ machines and FMMS by running them in at the manufacturers works[J]. Soviet Engineering Research, 1989, 9(3): 114-115.

[3] VASILEVV S. Cost efficiency approach to the selection of NC-machine tool reliability standards[J]. Soviet Engineering Research, 1985, 5(9): 49-50.

[4] VERSHININ B V, SHARIN Y S. Ways of increasing the reliability of NC equipment[J]. Soviet Engineering Research, 1987, 7(5): 71 .

[5] KELlER A $\mathrm{Z}$, KAMATHA $\mathrm{R} \mathrm{R}$, PERERA U D. Reliability analysis of CNC machine tools[J]. Reliability Engineering, 1982, 3(6): 449-473.

[6] GUPTA A, LAWSIRIRAT C. Strategically optimum maintenance of monitoring-enabled multi-component system using continuous-time jump deterioration models[J]. Journal of Quality in Maintenance Engineering, 2006, 12(3): 306-329.

[7] ARTSR H P M, SAXENAA, KNAPPG M. Estimation of distribution parameters of mixed failure mode data[J]. Journal of quality in maintenance engineering, 1997, 3(2): 120-135.

[8] KUO W, PRASAD V R. An annotated overview of system-reliability optimization[J]. IEEE Transactions on reliability, 2000, 49(2): 176-187.

[9] FREIHEIT T, HU S J. Impact of machining parameters on machine reliability and system productivity[J]. Journal of manufacturing science and engineering, 2002, 124(2): 296-304.

[10] MCGOLDRICK P F, KULLUK H. Machine tool reliability a critical factor in manufacturing systems [J]. Reliability Engineering, 1986, 14(3): 205-221.
[11] KHODABANDEHLOO K, SAYLES R S. Production reliability in flexible manufacturing systems[J]. Quality and Reliability Engineering International, 1986, 2(3): 171-182.

[12] MIRIYALA K, VISWANADHAM N. Reliability analysis of flexible manufacturing systems[J]. International Journal of Flexible Manufacturing Systems， 1989， 2(2): 145-162.

[13] YUANDIS P, STYBLINSKI M A, SMITH D R. Reliability modeling of flexible manufacturing systems[J]. Microelectronics and Reliability, 1994, 34(7): 1203-1220.

[14] CHAKRABORTY S, ANKIAH B. Assessment of manufacturing system reliability: A case study[J]. Journal of the Operational Research Society, 1989, 40(1): 55-63.

[15] WANG Y, YAM R C M, ZUO M J. A comprehensive reliability allocation method for design of $\mathrm{CNC}$ lathes[J]. Reliability Engineering \& System Safety, 2001， 72(3): 247-252.

[16] HAN B T, ZHANG C B, SUN C S. Reliability analysis of flexible manufacturing cells based on triangular fuzzy number[J]. Communications in Statistics, 2006, 35(10): 1897-1907.

[17] SAVSAR M, ALDAIHANI M. Modeling of machine failures in a flexible manufacturing cell with two machines served by a robot[J]. Reliability Engineering \& System Safety, 2008, 93(10): 1551-1562.

[18] BRALL A, CARDNER L. Availability modeling for the application of manufacturing equipment $[\mathrm{C}] / /$ Proceedings Annual Reliability Maintainability Symposium. [s. 1. ]: Institute of Electrical and Electronics Engineers Inc, 2002: 411-416.

[19] GÖRKEMLI L, ULUSOY S K. Fuzzy Bayesian reliability and availability analysis of production systems[J]. Computers \& Industrial Engineering, 2010, 59(4): 690-696.

[20] LIN Y K, CHANG P C. Performance indicator for a stochastic-flow manufacturing network with reworking actions based on reliability[J]. Scientia Iranica , Transaction E: Industrial Engineering, 2013, 20(6E2): 2201-2214.

[21] LIN Y K, HUANG C F, CHANG P C. System reliability evaluation of a touch panel manufacturing system with defect rate and reworking[J]. Reliability Engineering \& System Safety, 2013, 181: 51-60.

[22] LI J, MEERKOV S M. Customer demand satisfaction in 
production systems: A due-time performance approach[J]. IEEE Transactions on Robotics \& Automation, 2002, 17(4): 472-482.

[23] SAVSAR M. Modeling and simulation of a serial production line with constant[J]. Proceedings of World Academy of Science Engineering \& Technolog, 2011(53): 1195.

[24] AN J, DAI W, ZHAO Y. Reliability modeling for manufacturing process[C]// Prognostics \& System Health Management Conference, 23-25 May, Beijing, IEEE, 2012: 1-4.

[25] GARG H, SHARMA S P. Multi-objective reliability redundancy allocation problem using particle swarm optimization[J]. Computers \& Industrial Engineering, 2013, 64(1): 247-255.

[26] WU C H, DOUGLAS G DOWN, MARK E LEWIS. Heuristics for allocation of reconfigurable resources in a serial line with reliability considerations[J]. IIE Transactions, 2008, 40(6): 595-611.

[27] CONWAY R, MAXWELL W, MCCLAIN J O. The role of work-in-process inventory in serial production lines [J]. Operations Research, 1988, 36(2): 229-241.

[28] HANDFIELD R B, PANNESI R T. An empirical study of delivery speed and reliability[J]. International Journal of Operations \& Production Management, 1992, 12(2): 58-72.

[29] EMREENGINARLAR, JINGSHAN LI, MEERKOV S. Buffer capacity for accommodating machine downtime in serial production lines[J]. International Journal of Production Research, 2002, 40(3): 601-624.

[30] KOREN Y, HU S J, WEBER T W. Impact of manufacturing system configuration on performance[J]. CIRP Annals -Manufacturing Technology, 1998, 47(1): 369-372.

[31] SHI J. Stream of variation modeling and analysis for multistage manufacturing processes $[\mathrm{M}]$, Boca Raton: CRC Press, 2006.

[32] 沙基昌, 武小悦, 郭波. FMS 可靠性研究工作回顾 [J]. 国 防科技参考, 1995，16(2): 1-5.

SHA Jichang, WU Xiaoyue, GUO Bo. Review of FMS reliability research[J]. National Defense Science and Technology Reference, 1995, 16(2): 1-5.

[33] 胡华平. 柔性制造系统多任务可靠性建模与分析[D]. 长沙: 国防科学技术大学, 1995.

HU Huaping. Multi task reliability modeling and analysis of FMS [D]. Changsha: National University of Defense Science and Technology, 1995.

[34] 陈艳霞. 柔性制造系统可靠性形式化建模与分析 [J]. 电脑知识与技术，2012，8(1): 109-111.

CHEN Yanxia. Reliability formal modeling and analysis of the flexible manufacturing system[J]. Computer Knowledge and Technology, 2012, 08(1): 109-111.

[35] 高振清, 孙厚芳, 吴琼. 基于 GO 法的制造系统可靠性 分析[J]. 机械科学与技术, 2007, 26(3): 320-323.

GAO Zhenqing, SUN Houfang, WU Qiong. Reliability analysis of the manufacturing system based on GO methodology $[\mathrm{J}]$. Mechanical Science and Technology, 2007, 26(3): 320-323.

[36] 周丰旭, 李爱平, 李益, 等. 基于状态熵的多态制造系 统可靠性分析[J]. 计算机集成制造系统，2016，22(4): 1070-1078.

ZHOU fengxu, LI Aiping, LI Yi, et al. Reliability analysis of multi-state manufacturing system based on state entropy [J]. Computer Integrated Manufacturing System, 2016, 22(4): 1070-1078.

[37] 杨林. 串行制造系统可靠性研究与产能评估[D]. 成都: 西南交通大学, 2016.

YANG Lin. The reliability research and capacity Assessment of serial manufacturing system[D]. Chengdu : Southwest Jiaotong University, 2016.

[38] 柳剑. 制造系统运行可靠性分析与维修保障策略研究 [D]. 重庆: 重庆大学, 2014.

LIU Jian. Study on operational reliability analysis and maintenance system[D]. Chongqing: Chongqing University, 2014.

[39] 李娟. 面向制造过程的质量与可靠性联合优化 [D]. 重 庆：重庆大学， 2017.

LI Juan. Research on Joint optimization of quality and reliability for manufacturing process[D]. Chongqing: Chongqing University, 2017.

[40] 胡毓婷. 变速箱壳体生产线的可靠性分析与评价 [D]. 长春: 吉林大学, 2008.

HU Yuting. Reliability analysis and evaluation of gearbox shell production line[D]. Changchun: Jilin University, 2008.

[41] 丛中蔚. 生产系统可靠性分配方法与案例研究[D]. 长 春：吉林大学, 2014.

CONG Zhongwei. Method and case study research on production system reliability allocation [D]. Changchun: Jilin University, 2014.

[42] ZHANG Ding, ZHANG Yingjie, YU Mingrang, et al. 
Reliability evaluation and component importance measure

for manufacturing systems based on failure losses[J]. Journal of Intelligent Manufacturing, 2017, 28: 1859-1869.

[43] 匡芬, 戴伟, 陈亮, 等. 基于质量损失的加工过程可靠 性评估方法[J]. 计算机集成制造系统，2015，21(06): 1571-1578.

KUANG Fen, DAI Wei, CHEN Liang, et al. Assessment method and application for reliability manufacturing process based on quality loss[J]. Computer Integrated Manufacturing System, 2015, 21(06): 1571-1578.

[44] 匡芬, 戴伟, 王健, 等. 基于质量特性演化的工艺可靠 性评估 [J]. 计算机集成制造系统，2015，21(8)： 2124-2131.

KUANG Fen, DAI Wei, WANG Jiang, et al. Process reliability evaluation based on quality characteristics evolution[J]. Computer Integrated Manufacturing System, 2015, 21(8): 2124-2131.

[45] 付桂翠, 上官云, 史兴宽, 等. 基于产品可靠性的工艺 系统可靠性模型[J]. 北京航空航天大学学报, 2009, 35(1): 9-12.

FU Guicui, SHANGGUAN Yun, SHI Xingkuan, et al. Process system reliability model based on product reliability[J]. Journal of Beijing University of Aeronautics and Astronautics, 2009, 35(1): 9-12.

[46] 徐锐, 杜世昌, 任斐, 等. 基于马尔科夫模型的关联多 工序系统加工质量分析 $[\mathrm{J}]$. 上海交通大学学报, 2015, 49(5): 669-674.

XU Rui, DU Shichang, REN Fei, et al. Quality analysis of correlated multi-stage manufacturing systems based on markov model[J]. Journal of Shanghai Jiaotong University, 2015, 49(5): 669-674.

[47] 宋婷婷, 赵子任, 杜世昌, 等. 基于马尔科夫模型的关 联多工序装配系统装配质量分析 $[\mathrm{J}]$. 上海交通大学学 报，2018，52(3): 324-331.

SONG Tingting, ZHAO Ziren, DU Shichang, et al. Quality analysis of multi-stage assembly systems based on Markov model [J]. Journal of Shanghai Jiaotong University, 2018, 52(3): 324-331.

[48] 邓超, 吴军, 毛宽民, 等. 面向大型数控机床的工艺可 靠性评估[J]. 计算机集成制造系统，2010，16(10): 2250-2256.

DENG Chao, WU Jun, MAO kuanmin, et al. Process reliability evaluation for large-sized NC machine tools [J]. Computer integrated manufacturing system, 2010,
16(10): $2250-2256$

[49] 张根保, 葛红玉, 刘佳, 等. 可靠性驱动的装配过程 建模及预测方法[J]. 计算机集成制造系统, 2012, 18(2): 349-355.

ZHANG Genbao, GE Hongyu, LIU Jia, et al. Assembly process modeling and prediction method of reliability driven [J]. Computer Integrated Manufacturing System, 2012, 18(2): 349-355.

[50] CHEN Y, JIN J. Quality reliability chain modeling for system reliability analysis of complex manufacturing processes[J]. IEEE Transactions on Reliability, 2005, 54(3): 475-488.

[51] SUN J, Xi L, PAN E. Integration of product quality and tool degradation for reliability modelling and analysis of multi station manufacturing systems $[\mathrm{J}]$. International Journal of Computer Integrated Manufacturing, 2009, 22(3): 267-279.

[52] 吴军, 邓超, 邵新宇, 等. 基于加工动力学模型的工艺 可靠性仿真方法 $[\mathrm{J}]$. 系统工程理论与实践, 2011, 31(7): 1403-1408.

WU Jun, DENG Chao, SHAO Xinyu, et al. A dynamic model-based process reliability simulation method[J]. System Engineering-Theory and Practice, 2011, 31(7): 1403-1408.

[53] 杜彦斌，李聪波，刘世豪. 基于 GO 法的机床再制造工 艺过程可靠性分析方法[J]. 机械工程学报, 2017, 53(11): 203-210.

DU Yanbin, LI Congbo, LIU Shihao. Reliability assessment method of remanufacturing process for machine tools based on GO method[J]. Journal of Mechanical Engineering, 2017, 53(11): 203-210.

[54] JIANG Zhibin, HE Junming. Stochastic object-oriented petri nets(SPONS) and its application in modeling of manufacturing system reliability[J]. Chinese Journal of Mechanical Engineering, 2003， 16(3): 272-276.

[55] MACIEJ CHLEBUS, SYLWIA WERBIŃSKA WOJCIECHOWSKA. Assessment methods of production processes reliability-state of the art[J]. Journal of KONBiN，2017, 41: 247-276.

[56] HIMMELBLAU D M. Fault detection and diagnosis in chemical and petrochemical process[M]. Amsterdam: Elsevier Press, 1978.

[57] 孔繁森, 白小刚, 李从双, 等. 考虑人误的人机干涉排队 网络模型研究[J]. 机械工程学报, 2019, 55(14): 215-224. KONG Fansen, BAI Xiaogang, LI Congshuang, et al. 
Research of queuing network modelling of human-machine interference by considering human error[J]. Journal of mechanical engineering, 2019, 55(14): 215-224.

[58] KONG Fansen, ZHOU Yu, CHEN Taibo. Influence of man machine ratio on production line performance of one person multi machine series production line[C]// CIE49 Proceedings, October 18-21, Beihang University, 2019: 143.

[59] LI J, MEERKOV S M, ZHANG L. Production systems engineering: Main results and recommendations for management[J]. International Journal on Production
Research, 2013, 51: 7209-7234.

[60] LI J, MEERKOV S M. Production systems engineering, Berlin: Springer, 2009.

[61] GERSHWIN S B. Assembly/disassembly systems: An efficient decomposition algorithm for tree-structured networks. IIE Transactions, 1991, 23(4): 302-314.

作者简介: 孔繁森, 男, 1965 年出生, 教授, 博士研究生导师。主要研 究方向是制造系统的复杂性与可靠性、制造系统的设计评价、制造系统 建模中人的描述等。

E-mail: kongfs@jlu. edu.cn 